\section{By the Numbers}

\section{DAP: Digital Analytics Program}

Without big data analytics, companies are blind and deaf, wandering out onto the Web like deer on a freeway.

-Geoffrey Moore, Author of Crossing the Chasm and Inside the Tornado (twitter.com/ geoffreyamoore/status/234839087566163968)

E very day, millions of people access government websites. Over the thirty days preceding the date this article was written, the National Weather Service and the National Library of Medicine each received more than 50 million visits. ${ }^{1}$ According to the US General Services Administration, there are over 1,300 dot-gov domains in use by federal agencies. ${ }^{2}$ Given the immense resources involved in building and maintaining these websites, sound decisions about allocating these resources are important. And that means decision makers need good data.

In 2012, President Obama issued the Digital Government Strategy (purl.fdlp.gov/GPO/gpo23623). This strategy recognizes the power of technology and encourages government agencies to harness this power for the benefit of the American people. One of the many outcomes of this strategy is the Digital Analytics Program (DAP), which is housed in the General Services Administration. As of July 2015, the DAP "collects web traffic from around 400 executive branch government domains, across over 4000 total websites, including every cabinet department." ${ }^{3}$ This task is accomplished by means of a unified Google Analytics account used by the participating websites. ${ }^{4}$ The DAP framework promotes common government-wide performance measures for digital services, a common collection methodology, and a common web analytics tool.

I find this program fascinating on two fronts. First, the DAP produces a dashboard that highlights top usage of government websites. Second, the DAP provides resources for agencies to better work with their website analytics, and these resources can be easily applied by those outside the government who want to better work with the digital metrics for their own website.

\section{Web Analytics}

I presume that everyone reading this article has a basic understanding of web analytics, but I'll offer a simple description. Wikipedia defines web analytics as "the measurement, collection, analysis and reporting of web data for purposes of understanding and optimizing web usage." 5 The goal of web analytics is to understand more about how users interact with your web content so you can optimize your website and reduce inefficiencies. The following list describes just a few types of web analytics:

- Geographic analysis is one method of learning about the users of your website. Do they come from urban or rural areas? Or within a particular country, state, city, or county?

- Technology analysis may involve identifying whether website users access the site with a mobile or desktop device, a particular browser, operating system, and so on. This information can help you target your web design to meet the needs of your most frequent users.

- Page load speed can be used to evaluate a website's technical performance. Long page load times lead to low user satisfaction.

- Traffic source tells you how users arrived at your site and which marketing campaigns have been most successful at attracting them. Did they click a link on another website? Enter through a search engine result? Click a link in a newsletter?

- Search engine optimization reports can be used to identify the search terms people use to find your site, as well as the pages on your site that are most easily found through common web searches.

- Goals are fairly obvious for retail sites: the user makes a purchase. For noncommercial sites, goals may include the following:

- engagement goals (time on site, pages per visit, and so on)

- downloads

- newsletter subscriptions

- account registration

- clicks to social media accounts (Twitter, Facebook, and so on)

\section{DAP Dashboard}

The DAP dashboard (analytics.usa.gov) is a public display of highlighted web analytics collected from the participating dotgov websites. ${ }^{6}$ Users can also see the top dot-gov webpages currently being viewed, as well as the top domains (not specific webpages) over the past seven and thirty days. ${ }^{7}$ Usage breakdowns are also available by device, browser, operating system, and time of day — and all these data can be downloaded.

The dashboard shows high usage of weather service websites; currently seven of the top twenty pages are weather related (see figure 1). ${ }^{8}$ On the day this article was written, the New Horizons spacecraft was passing Pluto, pulling some related 


\section{By the Numbers}

NASA pages to the top of the list. Overall, much of the usage of government websites appears to be personal: What's my weather like? What's my case status? Where's my tax refund?

In addition to the aggregated metrics available on the DAP dashboard, some agencies are also publishing their own metrics. For example, the National Archives has its own dashboard (www.archives.gov/ metrics/).

The DAP dashboard is an open source project and is in the public domain. Interested parties can collaborate to make improvements or additions to the dashboard, or they can copy the code to build their own dashboard. ${ }^{9}$ The City of Philadelphia, for example, has built its own implementation of the dashboard (analytics.phila.gov).

\section{Useful Resources}

Google Analytics provides participating agencies with a tool that allows them to better understand their users and their websites. But simply providing the tool may not be enough - the agencies need to know how to use this tool. For this reason, resources for working with web analytics are provided at the DigitalGov website (www.digitalgov.gov/services/ dap). Although the site is targeted at government employees, DigitalGov's resources and expertise are shared with the public and can be useful to anyone who works with website analytics; use is not limited to participating federal agencies. Examples include a guide to best practices and tools relating to website analytics and metrics (www.digitalgov.gov/services/ dap/dap-digital-metrics-guidance-and-best-practices), a blog series (www.digitalgov.gov/category/metrics), and DigitalGov University training sessions. Anyone can sign up for a future session (www.digitalgov.gov/events) or view a recorded session (www.digitalgov.gov/digitalgov-university/video-library).

\section{Other Applications of the DAP Dashboard}

The DAP dashboard has the potential to be of great benefit to documents librarians. The dashboard states: "We plan to expand the data made available here. If you have any suggestions, or spot any issues or bugs, please open an issue on GitHub or contact the Digital Analytics Program." ${ }^{10}$ Personally, I would love to see more details. Usage by geographic area, by agency, or by site would all have great potential. ${ }^{11}$ This information could help documents librarians learn more about their users. For example, a regional depository coordinator could see what types of sites are accessed by users within their territory. Subject specialists could see the pages frequented by users within a particular field (economics, health, and so on). Perhaps by the time this column is published, some of these additions will have been made.

Pamela Campbell (pamela.d.campbell@stls.frb.org) is Senior Librarian, Federal Reserve Bank of St. Louis.

\section{References}

1. "Top Pages," accessed August 5, 2015, analytics.usa.gov.

2. "GAS/Data," GitHub, April 27, 2015, github.com/GSA/ data/blob/gh-pages/dotgov-domains/2015-03-15-federal.csv.

3. "About this Site," accessed July 13, 2015, analytics.usa.gov.

4. Not all agencies or websites have joined the program. The Pulse (pulse.cio.gov) depicts program participation by agency and by domain.

5. "Web Analytics," Wikipedia, accessed July 19, 2015, en.wikipedia.org/wiki/Web_analytics.

6. Staff at participating agencies have access to the full data through their Google Analytics accounts, but only highlights of the data are pushed to the public through the dashboard.

7. Since data are available only for the past thirty days, I went to the Internet Archive's Wayback Machine (web .archive.org/web/*/analytics.usa.gov) hoping to see the data for earlier dates. Unfortunately, because of how the page is built, the data displayed on an archived version of the page are identical to the data displayed on a live version of the page. 
8. The upcoming "By the Numbers" column will feature weather data and sources.

9. For a detailed overview of how the dashboard was put together, see "How We Built analytics.usa.gov" (18f.gsa .gov/2015/03/19/how-we-built-analytics-usa-gov).

10. "About this Site."

11. GovFresh blogger Luke Fretwell made some similar suggestions for expanding the use of these analytics in his
March 23, 2015, review of the dashboard shortly after its launch; see "Quick Thoughts, Takeaways from the New Federal Government Analytics Dashboard” (www.govfresh .com/2015/03/quick-thoughts-takeaways-from-the-new -federal-government-analytics-dashboard). 